This item was submitted to Loughborough's Research Repository by the author.

Items in Figshare are protected by copyright, with all rights reserved, unless otherwise indicated.

\title{
Equal sensation curves for whole-body vibration expressed as a function of
} driving force

PLEASE CITE THE PUBLISHED VERSION

http://dx.doi.org/10.1121/1.1898703

PUBLISHER

(C) Acoustical Society of America

VERSION

AM (Accepted Manuscript)

LICENCE

CC BY-NC-ND 4.0

\section{REPOSITORY RECORD}

Mansfield, Neil J., and Setsuo Maeda. 2019. "Equal Sensation Curves for Whole-body Vibration Expressed as a Function of Driving Force". figshare. https://hdl.handle.net/2134/11820. 
This item was submitted to Loughborough's Institutional Repository (https://dspace.lboro.ac.uk/) by the author and is made available under the following Creative Commons Licence conditions.

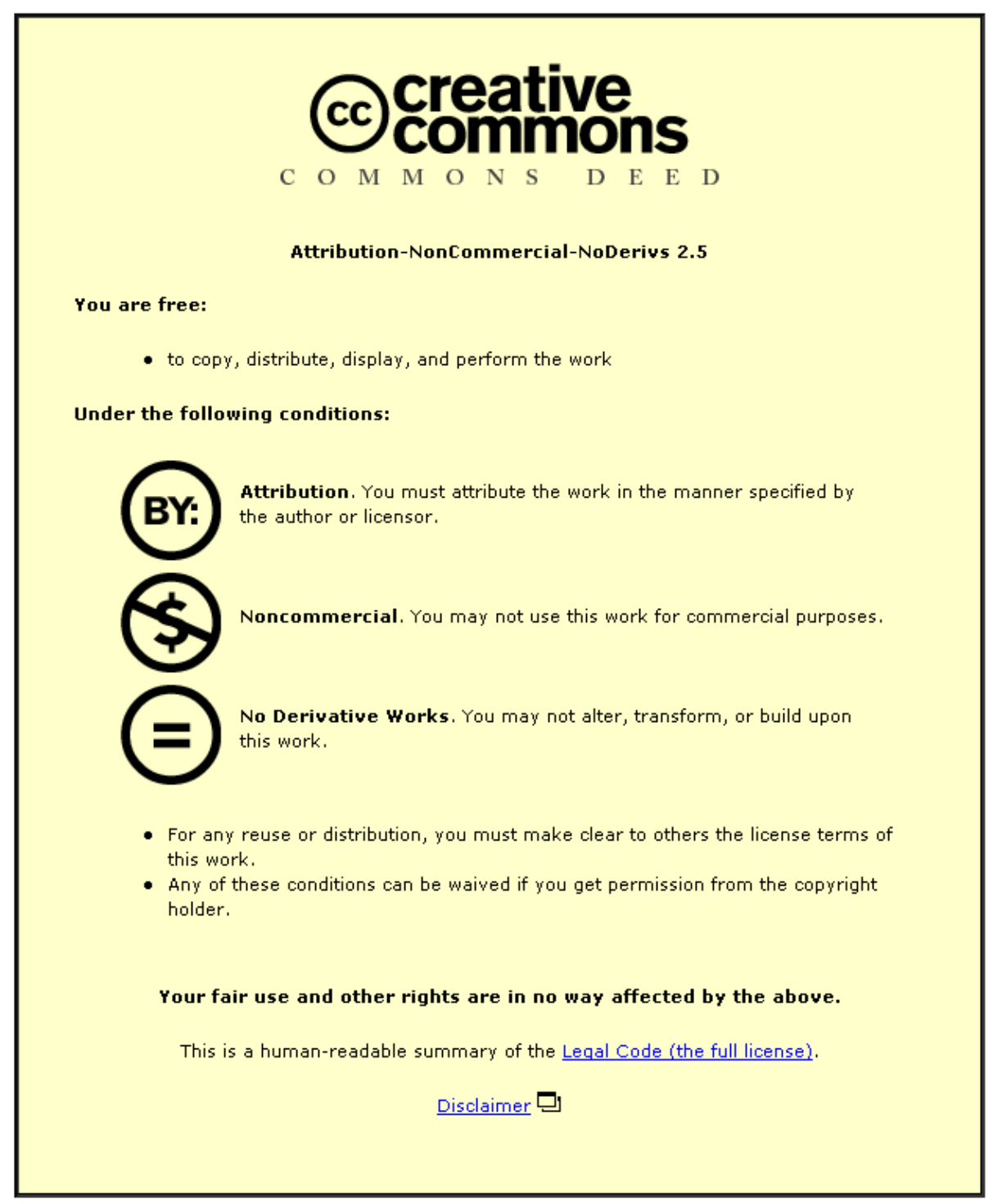

For the full text of this licence, please go to: http://creativecommons.org/licenses/by-nc-nd/2.5/ 
Equal sensation curves for whole-body vibration expressed as a function of driving force.

\author{
REVISED MANUSCRIPT
}

Dr Neil J Mansfield

Department of Human Sciences, Loughborough University, Loughborough, Leicestershire, LE11 3TU, UK

Tel: $\quad$ +44 1509228483

Email: n.j.mansfield@lboro.ac.uk

Dr Setsuo Maeda

Department of Human Engineering, National Institute of Industrial Health, 21-1, Nagao 6-Chome, Tama-Ku, Kawasaki 214-8585, Japan

Tel: $\quad+81-44-865-6111$

Email: maeda@niih.go.jp

Running title:

Perception of whole-body vibration force

\title{
43.66.Wv, 43.40.Ng
}

December 2004 


\section{ABSTRACT}

Previous studies have shown that the seated human is most sensitive to wholebody vertical vibration at about $5 \mathrm{~Hz}$. Similarly, the body shows an apparent mass resonance at about $5 \mathrm{~Hz}$. Considering these similarities between the biomechanical and subjective responses, it was hypothesised that, at low frequencies, subjective ratings of whole-body vibration might be directly proportional to the driving force.

Twelve male subjects participated in a laboratory experiment where subjects sat on a rigid seat mounted on a shaker. The magnitude of a test stimulus was adjusted such that the subjective intensity could be matched to a reference stimulus, using a modified Bruceton test protocol. The sinusoidal reference stimulus was $8 \mathrm{~Hz}$ vibration with a magnitude of $0.5 \mathrm{~m} / \mathrm{s}^{2}$ r.m.s. (or $0.25 \mathrm{~m} / \mathrm{s}^{2}$ r.m.s. for the $1 \mathrm{~Hz}$ test); the sinusoidal test stimuli had frequencies of $1,2,4,16$ and $32 \mathrm{~Hz}$.

Equal sensation contours in terms of seat acceleration showed data similar to those in the literature. Equal sensation contours in terms of force showed a nominally linear response at 1,2 and $4 \mathrm{~Hz}$ but an increasing sensitivity at higher frequencies. This is in agreement with a model derived from published subjective and objective fitted data. 


\section{INTRODUCTION}

Experimental work since the 1960s has indicated that perception of whole-body vibration is a function of vibration frequency (e.g. Miwa, 1969; Mansfield, 2004). Many studies have shown that the seated human is most sensitive to vertical vibration at about $4-5 \mathrm{~Hz}$ and most sensitive to horizontal vibration at lower frequencies (e.g. Parsons and Griffin, 1988). These data have been collated into a series of frequency weightings, such as those published in ISO2631 and BS6841 (e.g. Figure 1). These weightings model the subjective response to vibration such that, for single axis vibration:

$$
I(f) \propto a_{\text {weighted }}(f)
$$

where $I(f)$ is the subjective rating of vibration intensity and $a_{\text {weighted }}(f)$ is the frequency weighted acceleration at frequency $f$, although the relationship in terms of vibration magnitude is not necessarily linear (e.g. Morioka and Griffin, 2000).

\section{Figure 1 about here.}

The biomechanical response of the seated person has previously been assessed using kinetic (i.e. impedance and apparent mass) and kinematic (i.e. transmissibility) approaches. The apparent mass is calculated as the ratio of the force to the acceleration in the frequency domain:

$$
M(f)=\frac{F_{\text {seat }}(f)}{a_{\text {seat }}(f)}
$$


where $M(f)$ is the apparent mass, $F_{\text {seat }}(f)$ is the force and $a_{\text {seat }}(f)$ is the acceleration at frequency $f$ at the seat surface. There is a peak in the vertical apparent mass (or driving point mechanical impedance) of the seated human at about $5 \mathrm{~Hz}$ that reduces in frequency as the magnitude of the vibration increases. (e.g. Fairley and Griffin, 1989; Hinz and Seidel, 1987; Mansfield and Griffin, 2000; Smith 1994). The general shape of the peak is not dependent on vibration waveform or subject age, if the effects of subject mass are taken into account (Giacomin, 2004; Mansfield and Maeda, 2004). In the horizontal direction, a similar non-linearity has been observed, but with peaks in response at lower frequencies (Mansfield and Lundström, 1999a). The response of lumped parameter models (Mansfield and Lundström, 1999b) of the apparent mass of the seated human to whole-body vibration are shown in Figure 2.

\section{Figure 2 about here}

Considering the models of human responses to vibration depicted in Figures 1 and 2 it appears that subjective ratings of vibration and biomechanical responses might be related. For vertical motion, both subjective and biomechanical responses peak at about $5 \mathrm{~Hz}$. Similarly, for horizontal motion, both subjective and biomechanical responses peak at about $2 \mathrm{~Hz}$. However, for vertical vibration, the roll off at high frequencies for these models (and indeed for subject data) is not as great for the frequency weighting. 
The similarity between the subjective and biomechanical models at low frequency is sufficient to question whether there is an underlying link between the mechanical response (i.e. apparent mass) of the sitting person and their subjective response such that the apparent mass could effectively be used as a frequency weighting such that:

$$
I(f) \propto M(f) \times a_{\text {seat }}(f) .
$$

If this is the case then, considering the definition of apparent mass:

$$
I(f) \propto F_{\text {seat }}(f) .
$$

Therefore, subjective ratings of vibration intensity could be predicted solely from measurements of force at the seat. It should be noted that this technique would mean that no frequency weighting would be required for subjective assessments of vibration, and therefore it would be possible that inter-individual differences could be accounted for as an inherent property of the method. Furthermore, it would mean that non-linearities in response with respect to waveform and magnitude could be accounted for without the requirement for complex mathematical models. Although this approach is attractive, it is unclear whether it is viable either in terms of transducer technologies or whether the frequency range over which it might be effective is too restrictive. For example, the discrepancy between the apparent mass model and subjective response model could be less than or greater than that observed for individual subjects rendering the method more or less viable respectively. 
All known studies of human responses to vibration in the literature have scaled the dependent variable (biomechanical or subjective) in terms of the acceleration, velocity or displacement. To investigate whether human response to whole-body vibration is proportional to unweighted force, experimental data is required that links subjective ratings directly to driving force.

This paper reports a study that had the following objectives:

1. To define an equal comfort contour for whole-body vibration expressed in terms of force at the seat,

2. To identify the frequency range (if any) over which subjective rating is not a function of frequency when expressed in terms of force,

3. To provide a preliminary evaluation of the feasibility of using measurements of force at the seat as a predictor for subjective ratings of whole-body vibration. 


\section{METHODS}

A subjective intensity matching protocol was used in the experiment. Participants were exposed to five groups of trials. Each trial used a pair of sinusoidal vertical vibration stimuli: a reference stimulus at $8 \mathrm{~Hz}$ and a test stimulus with a frequency of either $1,2,4,16$ or $32 \mathrm{~Hz}$. $8 \mathrm{~Hz}$ was used for the reference to ensure that the reference and test stimuli frequencies were as close as possible when considered on a logarithmic scale (i.e. a maximum of three octaves) whilst avoiding the peak in apparent mass that could occur at $4 \mathrm{~Hz}$ for some subjects. Each stimulus had a duration of 4 seconds consisting of a 0.5 second taper, 3 seconds of steady sinusoidal vibration and finishing with a 0.5 second taper. There was a pause of 1 second between the reference and the test stimuli. For the $1 \mathrm{~Hz}$ trials, the reference stimulus (i.e. the $8 \mathrm{~Hz}$ stimulus) had a vibration magnitude of $0.25 \mathrm{~ms}^{-2}$; for all other trials, the reference stimulus had a vibration magnitude of $0.5 \mathrm{~ms}^{-2}$. The lower magnitude at $1 \mathrm{~Hz}$ was required to ensure that the experimental conditions remained within the specification of the vibrator.

Each trial used a modified Bruceton test protocol. After the pair of stimuli, subjects judged whether the first (reference) or second (test) stimulus had the greater intensity, or whether they felt the same. If the reference was perceived as being more intense, the magnitude of the test vibration was increased by $20 \%$. If the reference was perceived as being less intense, the magnitude of the test vibration was reduced by $20 \%$. A step size of $20 \%$ was used as this is approximately two times the difference threshold for whole-body vibration 
perception (e.g. Mansfield and Griffin, 2000; Morioka and Griffin, 2000). If the two stimuli were rated as being equal, then the magnitude of the test vibration was scaled by $20 \%$ in the same direction as the previous change. Therefore, the vibration magnitude would continually step up or down between a series of reversal points. The procedure was continued until six reversals had been completed (Figure 3). The value used for the matched intensity in the data analysis was the arithmetic mean of the six reversals for the acceleration and for the force. The starting magnitude for the test stimuli was randomly set at either 0.29 or $0.86 \mathrm{~ms}^{-2}$ r.m.s., $\mathrm{W}_{\mathrm{k}}$ weighted, apart from for the $1 \mathrm{~Hz}$ stimulus where these were set at 0.14 or $0.43 \mathrm{~ms}^{-2}$ r.m.s. Therefore, the first test stimulus should have been clearly perceived as either lower or higher than the reference stimulus respectively, assuming that the $W_{k}$ weighting is a reasonable model of wholebody vibration perception. The frequency weighting was only used to calculate the starting magnitude for each group of trials: all other reports of vibration magnitude in this paper are unweighted. The trial groups were presented in a balanced random order.

\section{Figure 3 about here}

Twelve male subjects participated in the experiment who were instructed to sit in a comfortable, relaxed upright posture. The subjects had a mean age of 27.9 years (s.d. 8.6), a mean weight of $65.8 \mathrm{~kg}$ (s.d. 11.6) and a mean stature of 171 cm (s.d. 11.6). 
An electro-hydraulic vibrator with low distortion was used to generate the vibration stimuli. This system had a peak-to-peak stroke of $100 \mathrm{~mm}$ and was situated in the laboratories of the National Institute of Industrial Health, Kawasaki, Japan. Acceleration at the seat was measured using a Brüel and Kjær 4370 accelerometer. The force at the seat was measured using a Kistler 9286A force plate which acted as the seat surface. The influence of the mass of the plate (approximately $15 \mathrm{~kg}$ ) was removed using a mass cancellation technique in the frequency domain. No backrest was used in the experiment. The vibrator was controlled by, and force and acceleration signals were acquired by, an HVLab data acquisition system at 512 samples per second via anti-aliasing filters set at $170 \mathrm{~Hz}$. Calculations were based on acquired acceleration magnitudes for the trial stimuli, rather than 'desired' vibration magnitudes. The control software included an algorithm to automatically equalise the next vibration stimulus based on the transfer function measured for the previous vibration stimulus. Measured vibration magnitudes remained within $5 \%$ of the desired magnitudes.

All subjects were explained the purpose of the study and gave their consent to participate. They were free to withdraw at any time although all subjects completed the trials. The experiment was approved by the Human Experimentation, Safety and Ethics Committee of the National Institute of Industrial Health. 


\section{RESULTS}

A full set of data was obtained for 11 of the 12 subjects. An equipment difficulty meant that data at $4 \mathrm{~Hz}$ was not obtained for one subject, although all other conditions were successfully measured. As ratings at $1 \mathrm{~Hz}$ were obtained relative to a reference with a magnitude of $50 \%$ of that used for all other conditions, measured values for acceleration and force were scaled by a factor of two to enable direct comparisons to be made.

For the acceleration data, most subjects showed that they were relatively less sensitive to the vibration at frequencies greater than the reference (i.e. test frequencies of 16 and $32 \mathrm{~Hz}$ ). At frequencies lower than the reference, most subjects also showed a slight reduction in sensitivity, but this was not observed for all individuals (Figure 4, Table I). Median data for the subjects expressed in terms of acceleration shows that there was not a clear difference between the sensitivities at 1 and $2 \mathrm{~Hz}$, sensitivities were slightly greater at $4 \mathrm{~Hz}$ and $8 \mathrm{~Hz}$ (the reference frequency) and that there was a steady decrease in sensitivity from 8 $\mathrm{Hz}$ through to $32 \mathrm{~Hz}$. The differences between the data obtained at $32 \mathrm{~Hz}$ and all other frequencies were significant $(p<0.005$, Wilcoxon). The only other significant difference occurred between the $2 \mathrm{~Hz}$ and $8 \mathrm{~Hz}$ data $(p<0.05)$ although differences approached significance between $1 \mathrm{~Hz}$ and $8 \mathrm{~Hz}(p=0.07)$ and between $8 \mathrm{~Hz}$ and $16 \mathrm{~Hz}(p=0.08)$.

\section{Figure 4 and Table I about here}


Due to the differences in the subject masses, the forces for the reference stimuli varied, despite the acceleration magnitude being controlled. Data were therefore normalised by dividing by the force at the reference magnitude to enable comparison between subjects (Table II). For the force data, all subjects showed that they were more sensitive to higher frequency forces than lower frequency forces. At frequencies above the reference $(8 \mathrm{~Hz})$, all subjects showed an increase in sensitivity (Figure 5). At frequencies below the reference, subjects tended to be slightly less sensitive in terms of force. All subjects were less sensitive to the vibration at $2 \mathrm{~Hz}$ and $4 \mathrm{~Hz}$ when compared to $8 \mathrm{~Hz}$, and ten of the subjects were less sensitive to vibration at $1 \mathrm{~Hz}$ when compared to $8 \mathrm{~Hz}$ (i.e. a greater force was required for parity). Most subjects showed a relatively equal response at $1 \mathrm{~Hz}, 2 \mathrm{~Hz}$ and $4 \mathrm{~Hz}$. The forces measured at $16 \mathrm{~Hz}$ and $32 \mathrm{~Hz}$ were all significantly lower than data obtained at lower frequencies $(p<0.005$, Wilcoxon) although there was no significant difference between the ratings at 16 $\mathrm{Hz}$ and $32 \mathrm{~Hz}$. Similarly, significant differences were observed between the data at $8 \mathrm{~Hz}$ and $1 \mathrm{~Hz}(\mathrm{p}<0.05)$ and between $8 \mathrm{~Hz}$ and $2 \mathrm{~Hz}$ or $4 \mathrm{~Hz}(\mathrm{p}<0.005)$. There were no significant differences between the results obtained at $1 \mathrm{~Hz}, 2 \mathrm{~Hz}$ and 4 $\mathrm{Hz}$.

Figure $\mathbf{5}$ and Table II about here 


\section{DISCUSSION}

This study used a single reference frequency of $8 \mathrm{~Hz}$. It is possible that the proximity of the reference and test frequencies could affect subjective responses; this could be investigated in future research. Unlike some previously published studies, the experiment did not use a binary forced choice protocol but a modified Bruceton protocol as described in the methods. The reason for this was to maintain the confidence of the subjects whilst making their judgements. Anecdotes from subjects used by the authors in previous experiments have suggested that participants feel uncomfortable being forced to 'guess' when performing forced-choice protocols and it was suspected that this could have contributed to occasional loss of concentration of some subjects during previous studies. A further advantage of the protocol used here is that it does not rely on interpretation of probabilities of chance 'correct' answers as occurs for forced choice up-and-down method of limits techniques (Maeda and Griffin, 1995). The choice of experimental protocol could have affected the results, although this is likely to have been a systematic difference and therefore not alter the relative ratings, as were sought in this experiment. Nevertheless, as results from this study are within the range of those in the literature, all indications are the methodology can be considered valid.

The equal sensation data in terms of acceleration produced in this experiment is comparable to other studies in the literature which have previously been identified as having minimal bias (Griffin et al. 1982). If published data sets are 
scaled to give a value of 0.5 at $8 \mathrm{~Hz}$, then the trends are similar between studies (Figure 6). All show frequencies of greatest sensation to occur between 4 and 8 $\mathrm{Hz}$ and a reduced sensation at higher and lower frequencies. The gradient of the reduction in sensation with increasing proximity from the 4-8 $\mathrm{Hz}$ range shows some variation between studies. Gradients obtained in this study were not as steep as some of those observed previously.

\section{Figure 6 about here}

The most commonly used frequency weighting for whole-body vertical vibration is the $W_{k}$ weighting (ISO2631-1, 1997). At frequencies of $4 \mathrm{~Hz}$ and above, the results for acceleration in this study correspond closely to the $W_{k}$ curve (Figure 6). However, below $4 \mathrm{~Hz}$ the $\mathrm{W}_{\mathrm{k}}$ curve shows a more rapid reduction in sensitivity than observed here. In agreement with the literature, the ratings of vibration intensity in this study had a discrepancy of approximately $2: 1$ at $1 \mathrm{~Hz}$ when compared to $W_{k}$. This indicates that $W_{k}$ might underestimate subjective ratings of vibration magnitude at low frequency. However, $W_{k}$ is also designed to be applicable for health risk assessments. Biomechanical data (e.g. Smith, 2000; Boileau et al., 2002; Nawayseh and Griffin, 2003) tend to show a resonance in the response of the seated body to vibration at about $5 \mathrm{~Hz}$. Therefore, data from this study alone does not necessarily imply that the frequency weighting curve should be changed (at least for health risk assessments). 
Previous studies have not generally reported subjective and biomechanical responses to whole-body vibration within the same experiment. Therefore, it is not possible to derive the relationship between any individual's subjective response to force and the frequency of the force. However, it is possible to use a model of subjective response (i.e. a frequency weighting) in combination with a model of biomechanical response (i.e. a lumped parameter apparent mass model) to derive a generalised expected profile for subjective responses with respect to force at any frequency. As apparent mass is defined as the ratio of the force to the acceleration at any frequency and the frequency weighting is modelled to the subjective rating of acceleration at any frequency, division of the apparent mass by the frequency weighting will result in a model of an equal sensation response in terms of force. A model derived from the $W_{k}$ weighting and the lumped parameter model presented in Mansfield and Lundström (1999b), where the data sets are scaled to give a value of unity at $8 \mathrm{~Hz}$, is shown in Figure 7 and compared to the data obtained in this study. This shows that at frequencies below $5 \mathrm{~Hz}$ the effect of frequency observed in the weighting and in the apparent mass cancel one another out such that the subjective response is linear with respect to frequency. At frequencies above $5 \mathrm{~Hz}$ the equal sensation curve decays indicating that a lower force is required for an equivalent sensation. Therefore, a greater sensation occurs for the same r.m.s. force.

\section{Figure 7 about here}


The similarity between the shape of the response of the model of an equal sensation curve for force based on fitted curves and the results obtained in this study is clear. Although in the representation illustrated in Figure 7 the differences between the curves occur at low frequency, this is due to the normalisation occurring at $8 \mathrm{~Hz}$; if the normalisation occurred at, for example, 1 $\mathrm{Hz}$, the difference would be observed at the higher frequencies. These data therefore indicate that the gradient of increasing sensation at high frequencies is steeper in the model than that observed in this study. The frequency at which the response starts to be affected by vibration frequency was similar for the model and the experimental data.

Although subjects were not interviewed in a structured manner following the trials, it was commented that the low frequency stimuli were perceived by the whole body whereas the sensations of high frequency vibration were localised to the skin and muscle of the thigh and buttocks. This corresponds to published data concerning transmission of vibration through the seated person which shows that sites remote from the driving point are effectively isolated from the vibration at higher frequencies (e.g. Paddan and Griffin, 1998). Therefore the mechanism for the increased sensitivity at high frequencies must either be due to the nature of the underlying physiology (e.g. fast adapting nerve endings in the skin sensitive to vibration above $10 \mathrm{~Hz}$ ) or an indirect result of the biomechanical response. The increased sensitivity to force at high frequencies being due to the biomechanical response is the most plausible as less force is required to 
generate the same acceleration at high frequencies when compared to low frequencies, as can be seen in the apparent mass of the seated body (Figure 2).

This experiment has indicated that for low frequency vibration, an assessment methodology based on driving force could be envisaged. However, the frequency range over which this would be of value would be constrained to frequencies below about $5 \mathrm{~Hz}$. Furthermore, there are practical difficulties in measuring the driving force in vehicles, although some studies have reported such data (e.g. Holmlund, 1999). Considering that most environments where individuals are exposed to vibration have a significant proportion of vibration energy at frequencies above this $5 \mathrm{~Hz}$ threshold, the use of force cannot be considered a pragmatic method of vibration assessment. 


\section{CONCLUSIONS}

This study has shown that the sensitivity of seated humans to whole-body vibration is greatest for acceleration at a frequencies between 4 and $8 \mathrm{~Hz}$. This is in agreement with the literature, although a slightly different psychophysical technique was used. Subjective ratings of vibration in terms of force showed that the greatest sensitivity occurred at high frequencies. A nominally linear response occurred between 1 and $4 \mathrm{~Hz}$. This is in agreement with a model of response based on a frequency weighting and apparent mass model in the literature. 


\section{ACKNOWLEDGEMENTS}

The authors acknowledge the assistance of staff and students at Tokyo Metropolitan University. 


\section{REFERENCES}

Boileau,PE; Rakheja,S and Wu, X (2002) A body mass dependent mechanical impedance model for applications in vibration seat testing. Journal of Sound and Vibration, 253(1) 243-264.

British Standards Institution (1987) Measurement and evaluation of human exposure to whole-body mechanical vibration and repeated shock. BS6841. BSI, London.

Fairley TE and Griffin MJ (1989) The apparent mass of the seated human body: vertical vibration. Journal of Biomechanics 22(2), 81-94.

Giacomin J (2004) Apparent mass of small children: experimental measurements. Ergonomics 47 (13), 1454-1474.

Griffin MJ, Parsons KC and Whitham EM (1982) Vibration and comfort IV. Application of experimental results. Ergonomics 25(8), 721-739.

Hinz B and Seidel H (1987) The nonlinearity of the human body's dynamic response during sinusoidal whole body vibration. Industrial Health 25, 169-181.

Holmlund, P (1999) Absorbed power and mechanical impedance of the seated human measured within a real vehicle environment compared with single axis laboratory data. Journal of Low Frequency Noise Vibration and Active Control, 18(3) 97-110. 
International Organization for Standardization (1997) Mechanical vibration and shock - evaluation of human exposure to whole-body vibration: ISO2631-1. ISO, Geneva.

Maeda S and Griffin MJ (1995) A comparison of vibrotactile thresholds on the finger obtained with different measuring algorithms. Proceedings of Stockholm Workshop 1994: Hand-Arm Vibration: Diagnostics and Quantitative Relationships to Exposure. Arbete och Halsa 1995.

Mansfield NJ (2004) Human response to vibration. CRC Press, ISBN 0-41528239-X.

Mansfield NJ and Griffin MJ (2000) Difference thresholds for automobile seat vibration. Applied Ergonomics 31(3) 255-261.

Mansfield NJ and Griffin MJ (2000) Non-linearities in apparent mass and transmissibility during exposure to whole-body vibration. Journal of Biomechanics 33(8), 933-941.

Mansfield NJ and Lundström R (1999a) The apparent mass of the human body exposed to non-orthogonal horizontal vibration. Journal of Biomechanics 32(12), 1269-1278.

Mansfield NJ and Lundström R (1999b) Models of the apparent mass of the seated human body exposed to horizontal whole-body vibration. Aviation Space and Environmental Medicine 70(12) 1166-1172.

Mansfield NJ and Maeda S (2004) Comparison of the apparent mass of the seated human measured using random and sinusoidal vibration. Industrial Health (in press). 
Miwa T (1969) Evaluation methods for vibration effect. Part 8: The vibration greatness of random waves. Industrial Health, 7, 89-115.

Morioka, M and Griffin, MJ (2000) Difference thresholds for intensity perception of whole-body vertical vibration : Effect of frequency and magnitude. Journal of the Acoustical Society of America, 107(1), 2000, 620-4.

Nawayseh,N and Griffin,MJ (2003) Non-linear dual-axis biodynamic response to vertical whole-body vibration. Journal of Sound and Vibration, 268(3) 503-523.

Paddan, GS and Griffin, MJ (1998) A review of the transmission of translational seat vibration to the head. Journal of Sound and Vibration 215(4), 863-882.

Parsons KC and Griffin MJ (1988) Whole-body vibration perception thresholds. Journal of Sound and Vibration, 121(2), 237-258.

Smith SD (1994) Nonlinear resonance behavior in the human exposed to wholebody vibration. Shock and Vibration, 1(5), 439-450.

Smith SD (2000) Modeling differences in the vibration response characteristics of the human body. Journal of Biomechanics, 33(11),1513-1516. 


\section{TABLES}

Table I. Individual data corresponding to equal sensation contours for rating of whole-body vibration in terms of acceleration.

\begin{tabular}{|c|cccccc|}
\hline \multicolumn{7}{|c|}{ Acceleration (m/s ${ }^{2}$ r.m.s.) } \\
\hline Frequency & $1 \mathrm{~Hz}$ & $2 \mathrm{~Hz}$ & $4 \mathrm{~Hz}$ & $8 \mathrm{~Hz}$ & $16 \mathrm{~Hz}$ & $32 \mathrm{~Hz}$ \\
\hline Subject 1 & 0.36 & 0.43 & 0.47 & 0.50 & 0.39 & 0.85 \\
Subject 2 & 0.42 & 0.56 & 0.61 & 0.50 & 0.69 & 0.55 \\
Subject 3 & 0.94 & 0.42 & 0.51 & 0.50 & 0.54 & 1.56 \\
Subject 4 & 0.44 & 0.56 & 0.42 & 0.50 & 0.81 & 1.01 \\
Subject 5 & 1.35 & 0.84 & 0.63 & 0.50 & 0.81 & 1.86 \\
Subject 6 & 0.83 & 0.66 & 0.49 & 0.50 & 0.46 & 0.85 \\
Subject 7 & 0.53 & 0.88 & - & 0.50 & 0.61 & 1.05 \\
Subject 8 & 0.70 & 0.63 & 0.40 & 0.50 & 0.39 & 1.06 \\
Subject 9 & 0.77 & 0.84 & 0.68 & 0.50 & 0.39 & 1.35 \\
Subject 10 & 0.66 & 0.51 & 0.51 & 0.50 & 0.78 & 1.44 \\
Subject 11 & 0.50 & 0.50 & 0.44 & 0.50 & 0.65 & 1.56 \\
Subject 12 & 0.52 & 0.69 & 0.70 & 0.50 & 0.67 & 1.01 \\
\hline $25^{\text {th }}$ percentile & 0.49 & 0.51 & 0.45 & 0.50 & 0.44 & 0.97 \\
Median & 0.60 & 0.60 & 0.51 & 0.50 & 0.63 & 1.05 \\
$75^{\text {th }}$ percentile & 0.78 & 0.73 & 0.62 & 0.50 & 0.72 & 1.47 \\
\hline
\end{tabular}


Table II. Individual data corresponding to equal sensation contours for rating of whole-body vibration in terms of r.m.s. normalised force.

\begin{tabular}{|c|cccccc|}
\hline \multicolumn{7}{|c|}{ Normalised force (r.m.s.) } \\
\hline Frequency & $1 \mathrm{~Hz}$ & $2 \mathrm{~Hz}$ & $4 \mathrm{~Hz}$ & $8 \mathrm{~Hz}$ & $16 \mathrm{~Hz}$ & $32 \mathrm{~Hz}$ \\
\hline Subject 1 & 0.855 & 1.102 & 1.387 & 1.000 & 0.338 & 0.168 \\
Subject 2 & 0.717 & 1.014 & 1.267 & 1.000 & 0.449 & 0.094 \\
Subject 3 & 2.856 & 1.382 & 2.133 & 1.000 & 0.437 & 0.632 \\
Subject 4 & 1.351 & 1.829 & 1.788 & 1.000 & 0.442 & 0.410 \\
Subject 5 & 5.118 & 3.606 & 4.551 & 1.000 & 0.509 & 0.658 \\
Subject 6 & 3.162 & 2.689 & 2.755 & 1.000 & 0.361 & 0.467 \\
Subject 7 & 2.119 & 3.879 & - & 1.000 & 0.676 & 0.379 \\
Subject 8 & 1.772 & 1.702 & 1.196 & 1.000 & 0.436 & 0.150 \\
Subject 9 & 2.036 & 2.361 & 2.293 & 1.000 & 0.372 & 0.479 \\
Subject 10 & 1.568 & 1.206 & 1.593 & 1.000 & 0.330 & 0.358 \\
Subject 11 & 1.005 & 1.085 & 1.176 & 1.000 & 0.401 & 0.331 \\
Subject 12 & 1.099 & 1.493 & 1.736 & 1.000 & 0.479 & 0.393 \\
\hline $25^{\text {th }}$ percentile & 1.076 & 1.180 & 1.327 & 1.000 & 0.369 & 0.290 \\
Median & 1.670 & 1.598 & 1.736 & 1.000 & 0.436 & 0.386 \\
$75^{\text {th }}$ percentile & 2.303 & 2.443 & 2.213 & 1.000 & 0.456 & 0.470 \\
\hline
\end{tabular}




\section{FIGURE CAPTIONS}

Figure 1. Modulus of frequency weightings for vertical vibration $\left(W_{k},-x-\right)$ and horizontal vibration $\left(\mathrm{W}_{\mathrm{d}}, \longrightarrow\right.$ ) as used in ISO2631-1 (1997).

Figure 2. Normalised responses of lumped parameter apparent mass models defined in Mansfield and Lundström, 1999.

Figure 3. Example of experimental protocol for one group of trials. After each rating of the test stimulus being more intense than the reference $(+)$, the test vibration magnitude decreased by $20 \%$. After each rating of the test stimulus being less intense than the reference (-), the test vibration magnitude increased by $20 \%$. If the stimuli were rated as equal (o), the test magnitude was stepped up or down, repeating the previous change. The arithmetic mean of the six reversals $(\square)$ was used to determine the magnitude of equal sensation for the reference and test stimuli for that group of trials (- - - ).

Figure 4. Equal sensation curves for 12 subjects exposed to whole-body vertical vibration. Data represent accelerations corresponding to three positive and three negative reversals at each frequency (o) and mean of all reversals at each frequency (-). 
Figure 5. Equal sensation curves for 12 subjects exposed to whole-body vertical vibration. Data represent the r.m.s. force corresponding to three positive and three negative reversals at each frequency (o) and mean of all reversals at each frequency $(\longrightarrow)$.

Figure 6. Comparison of median equal sensation acceleration data obtained in this study (—o—) with data from Miwa (1967; — - - —), Shoenberger and Harris (1971; - - - ), Jones and Saunders (1972; - $\longrightarrow)$, Griffin at al. (1982; - - -) and an inverted $W_{k}$ frequency weighting $(--)$, all scaled to give a value of 0.5 at $8 \mathrm{~Hz}$ (the reference frequency in this study).

Figure 7. Comparison of median equal sensation normalised force data obtained in this study (-•-) with interquartile ranges (- - -) and results from the model based on frequency weighting and biomechanical models in the literature (-), normalised at $8 \mathrm{~Hz}$. 


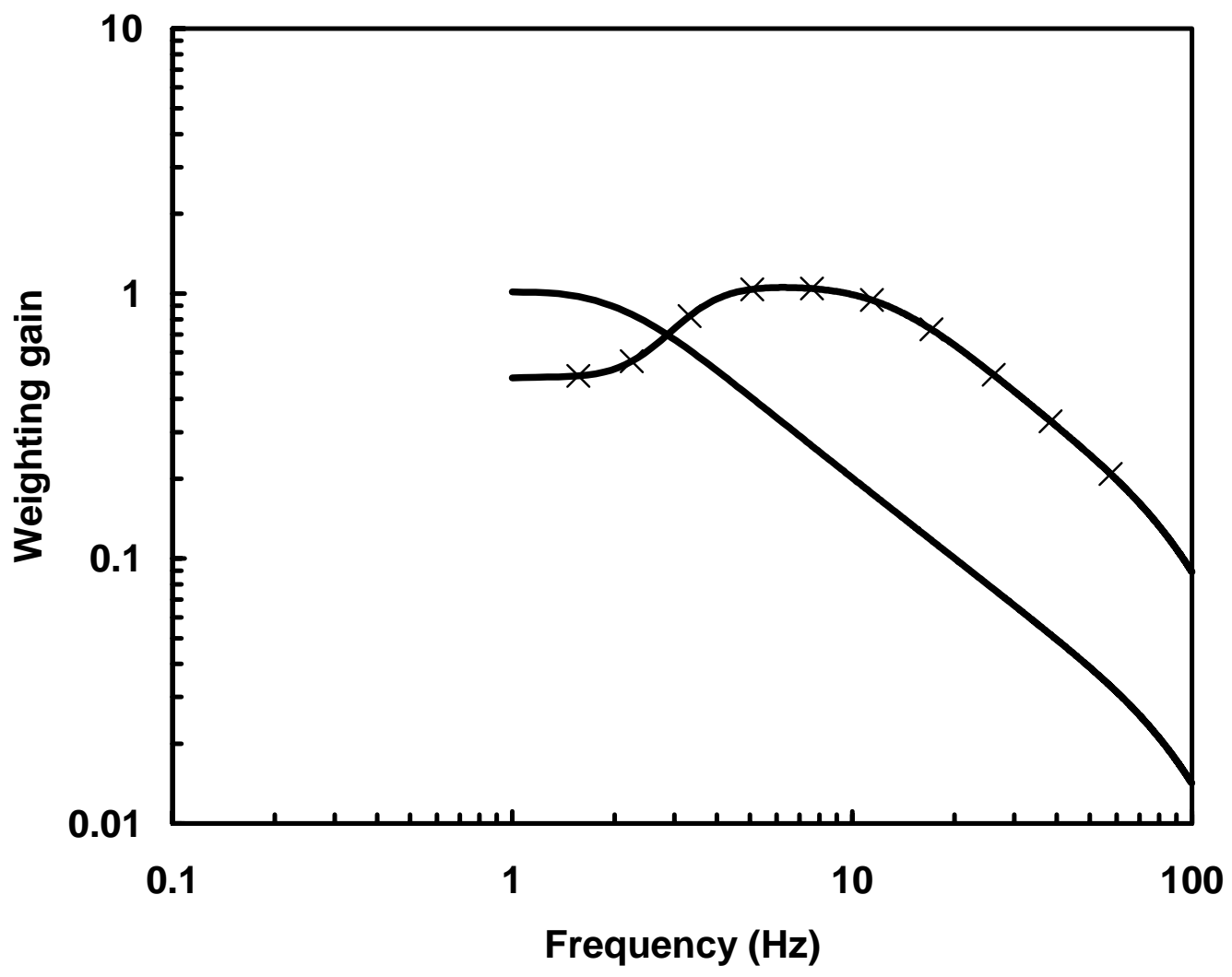

Figure 1. Modulus of frequency weightings for vertical vibration $\left(W_{k},-x\right)$ and horizontal vibration $\left(\mathrm{W}_{\mathrm{d}}, \longrightarrow\right.$ ) as used in ISO2631-1 (1997). 


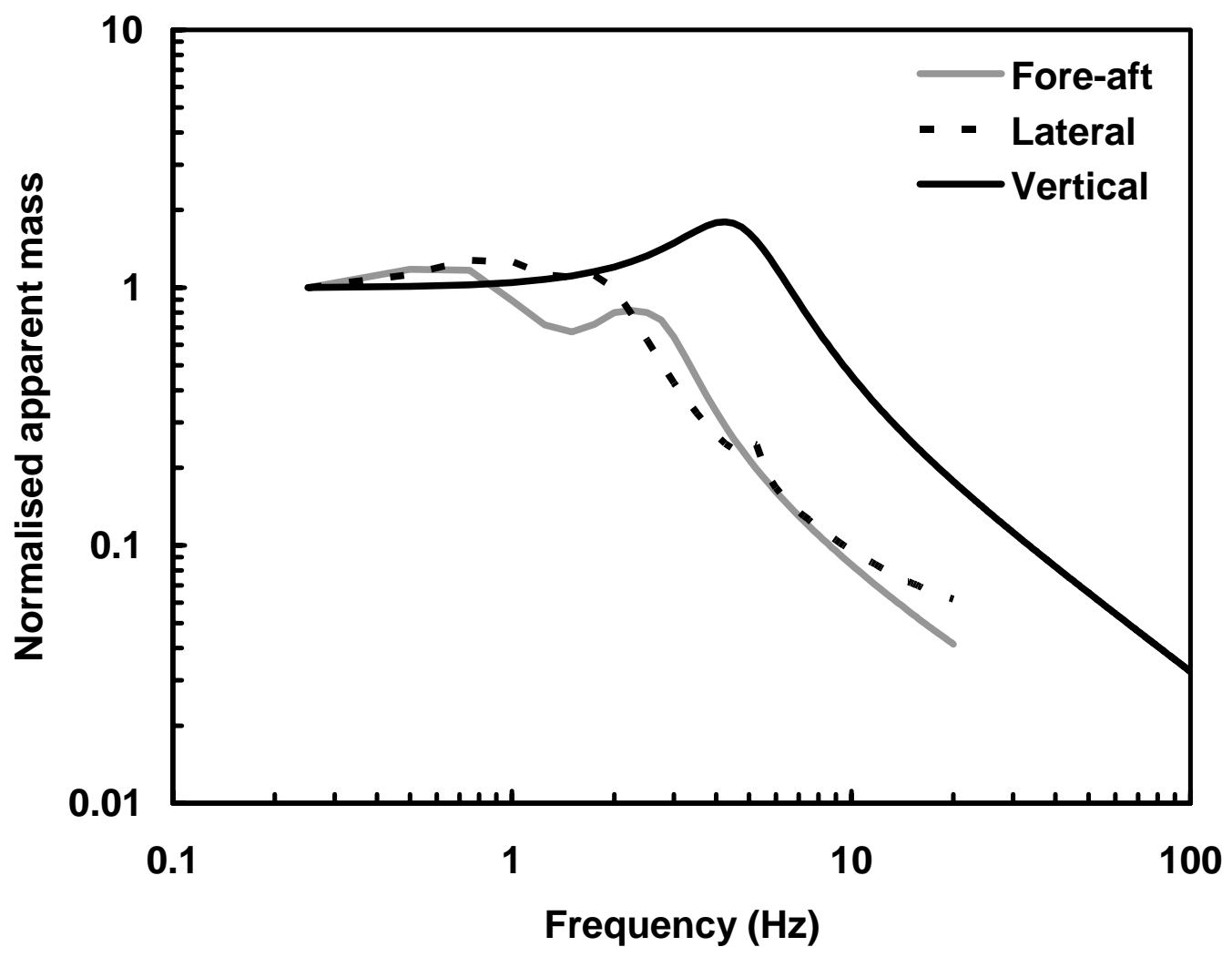

Figure 2. Normalised responses of lumped parameter apparent mass models defined in Mansfield and Lundström, 1999. 


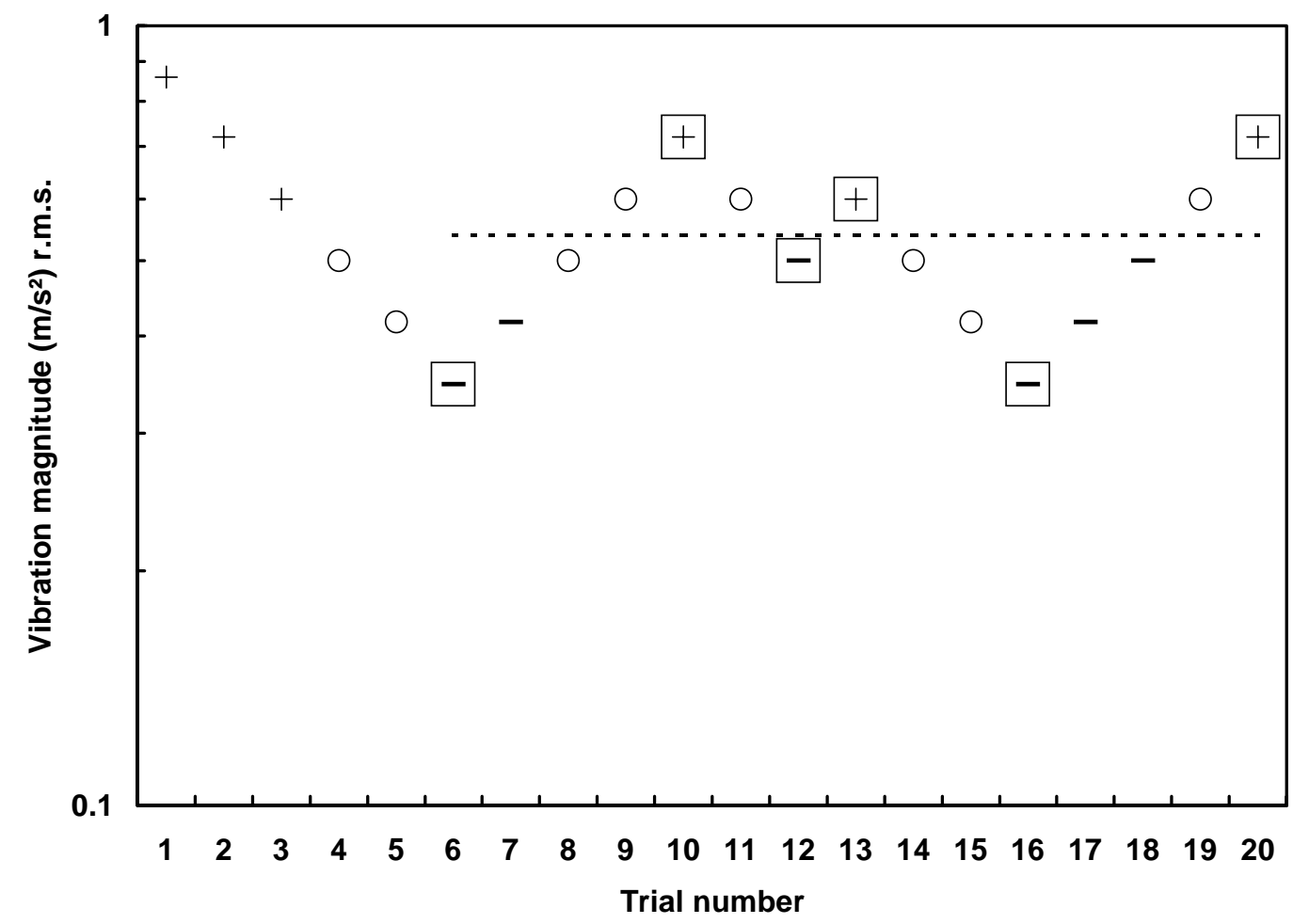

Figure 3. Example of experimental protocol for one group of trials. After each rating of the test stimulus being more intense than the reference $(+)$, the test vibration magnitude decreased by $20 \%$. After each rating of the test stimulus being less intense than the reference (-), the test vibration magnitude increased by $20 \%$. If the stimuli were rated as equal (o), the test magnitude was stepped up or down, repeating the previous change. The arithmetic mean of the six reversals ( $\square$ ) was used to determine the magnitude of equal sensation for the reference and test stimuli for that group of trials (- - - ). 

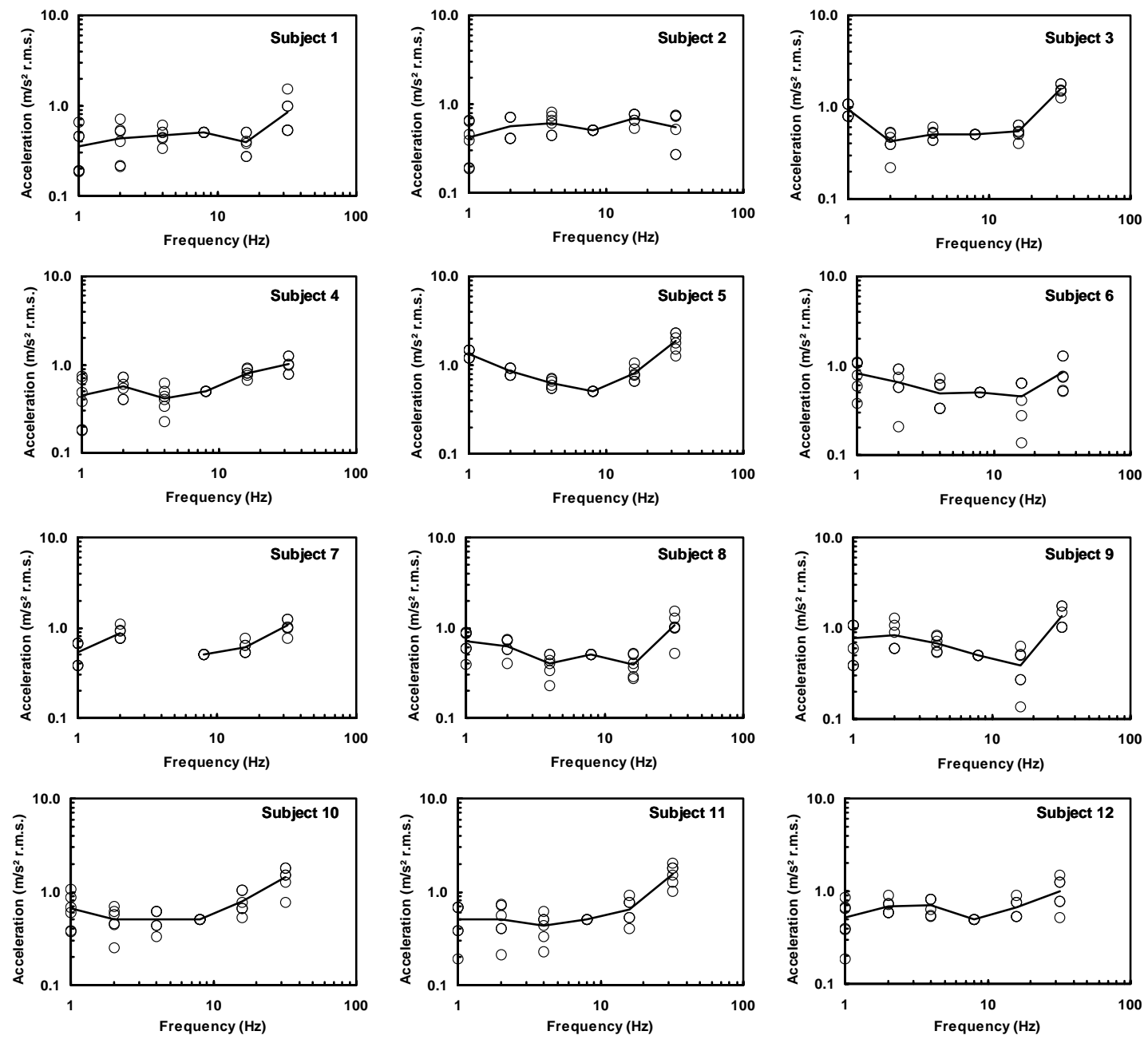

Figure 4. Equal sensation curves for 12 subjects exposed to whole-body vertical vibration. Data represent accelerations corresponding to three positive and three negative reversals at each frequency (o) and mean of all reversals at each frequency $(\longrightarrow)$. 

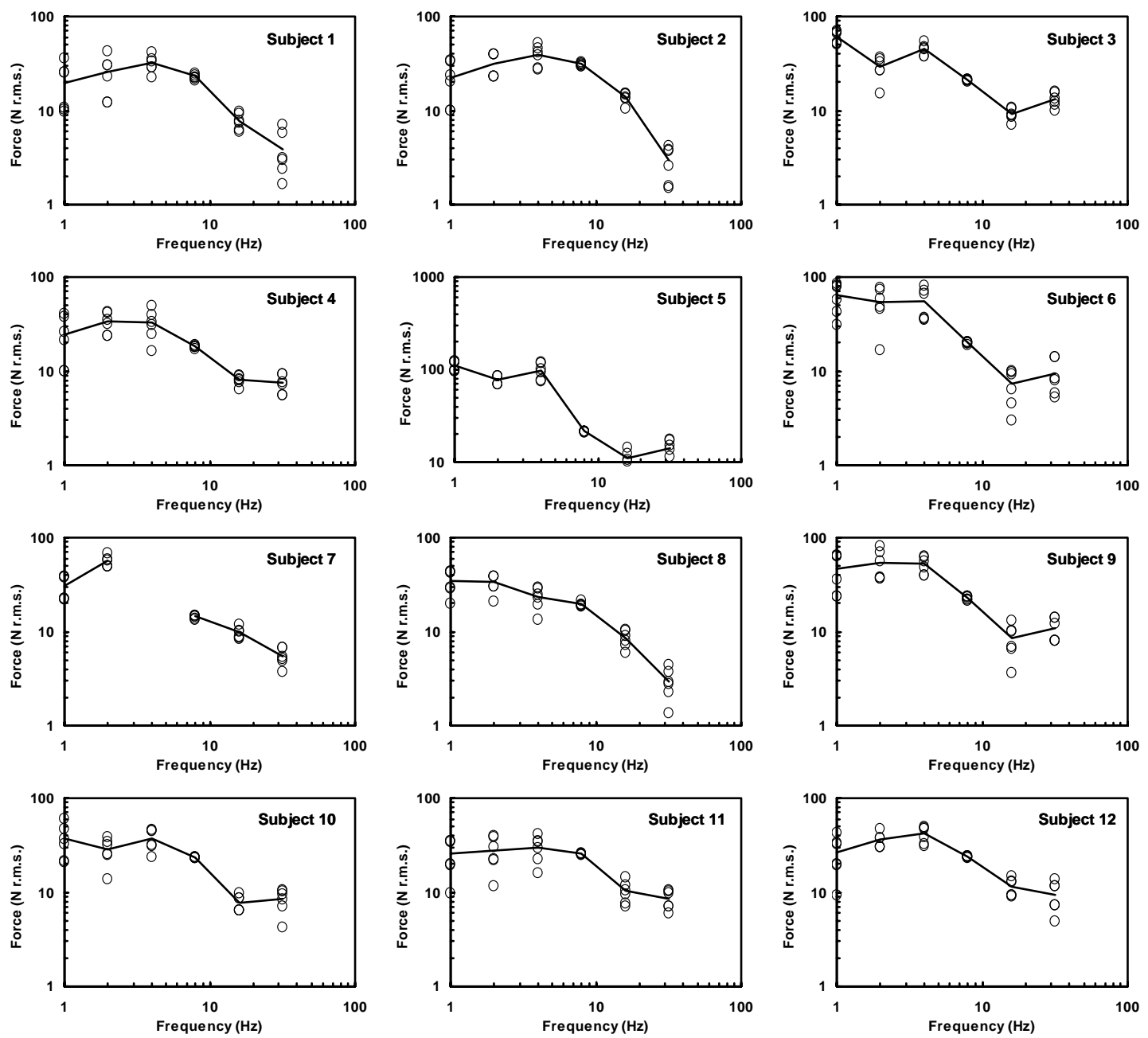

Figure 5. Equal sensation curves for 12 subjects exposed to whole-body vertical vibration. Data represent the r.m.s. force corresponding to three positive and three negative reversals at each frequency (o) and mean of all reversals at each frequency $(\longrightarrow)$. 


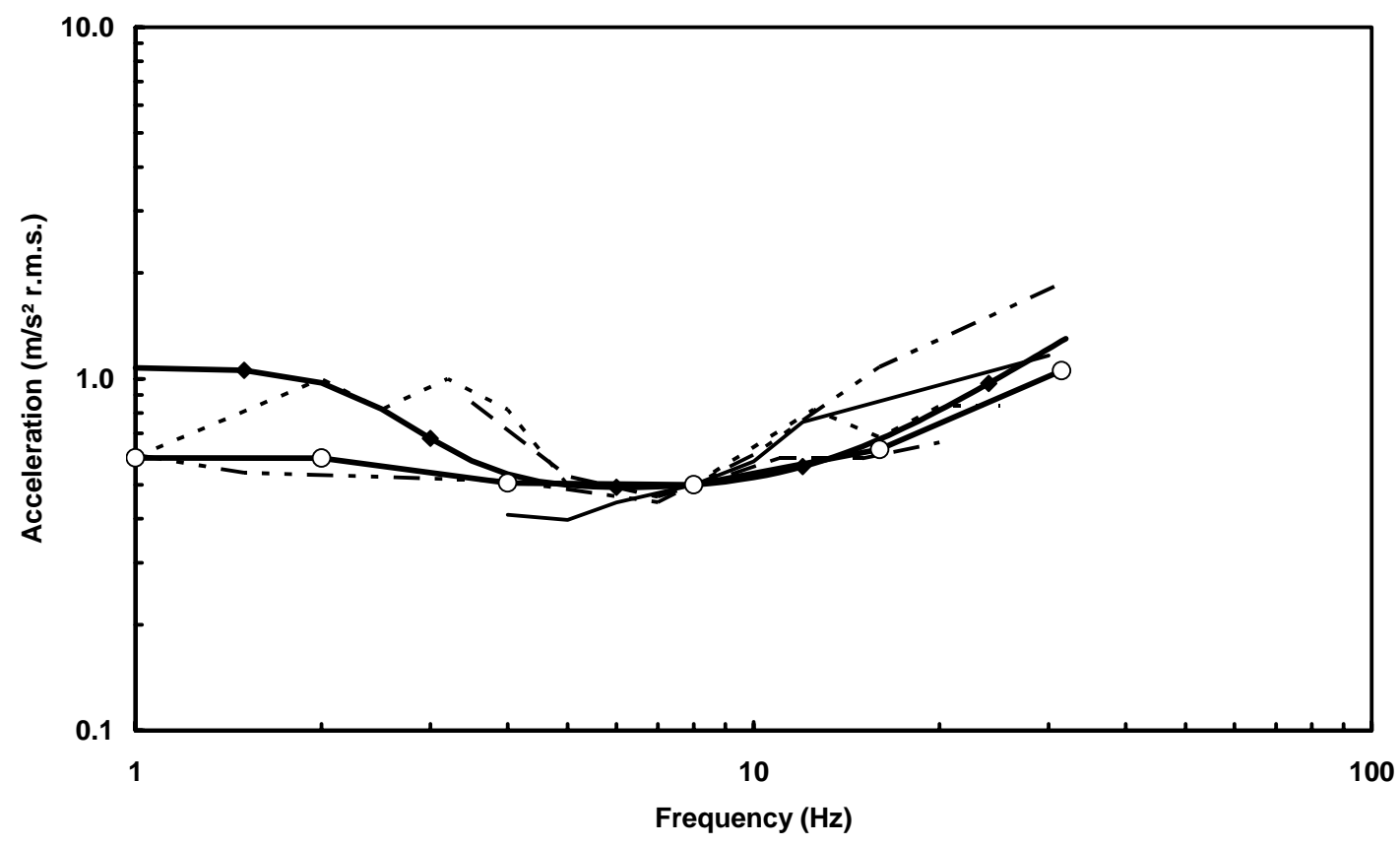

Figure 6. Comparison of median equal sensation acceleration data obtained in this study (—o—) with data from Miwa (1967; — - - -), Shoenberger and Harris $(1971 ;-\ldots-$ - $)$, Jones and Saunders $(1972 ;-)$, Griffin at al. (1982; - - -) and an inverted $W_{k}$ frequency weighting (- - ) all scaled to give a value of 0.5 at $8 \mathrm{~Hz}$ (the reference frequency in this study). 


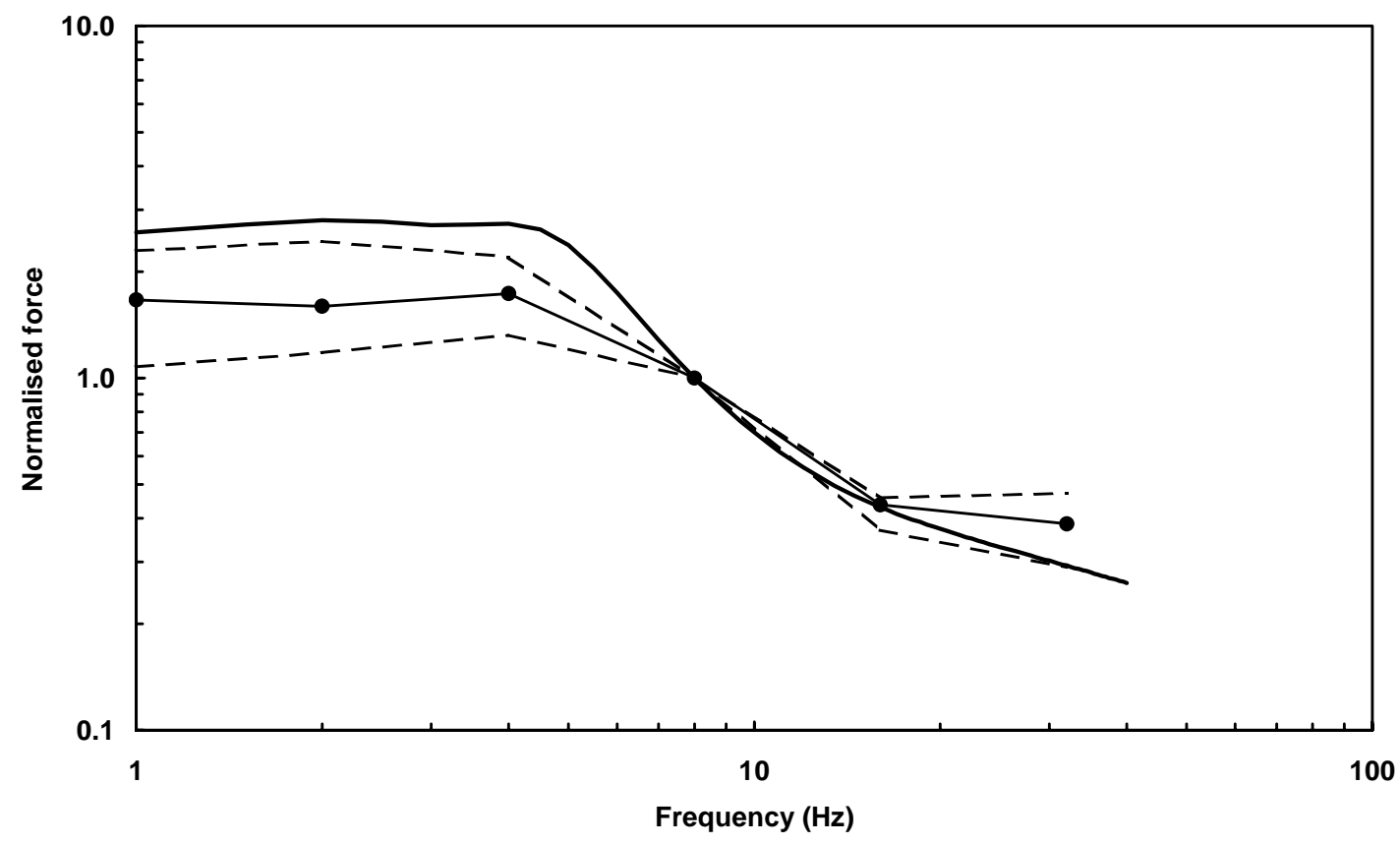

Figure 7. Comparison of median equal sensation normalised force data obtained in this study (-•-) with interquartile ranges (- - -) and results from the model based on frequency weighting and biomechanical models in the literature (-), normalised at $8 \mathrm{~Hz}$. 